\title{
Platelet function-guided modification in antiplatelet therapy after acute ischemic stroke is associated with clinical outcomes in patients with aspirin nonresponse
}

\author{
Xingyang $\mathrm{Yi}^{1}$, Jing Lin ${ }^{2}$, Chun Wang ${ }^{1}$, Ruyue Huang ${ }^{2}$, Zhao Han ${ }^{3}$ and $\mathrm{Jie} \mathrm{Li}^{1}$ \\ ${ }^{1}$ Department of Neurology, People's Hospital of Deyang City, Deyang 618000, Sichuan, China \\ ${ }^{2}$ Department of Neurology, The Third Affiliated Hospital of Wenzhou Medical University, Wenzhou 325200, Zhejiang, China \\ ${ }^{3}$ Department of Neurology, The Second Affiliated Hospital and Yuying Children's Hospital of Wenzhou Medical University, \\ Wenzhou 325027, Zhejiang, China
}

Correspondence to: Jing Lin, email: 1842942576@qq.com

Keywords: aspirin; ischemic stroke; resistance; platelet function testing; nonresponse

Received: June 15, $2017 \quad$ Accepted: October 25, $2017 \quad$ Published: November 07, 2017

Copyright: Yi et al. This is an open-access article distributed under the terms of the Creative Commons Attribution License 3.0 (CC BY 3.0), which permits unrestricted use, distribution, and reproduction in any medium, provided the original author and source are credited.

\section{ABSTRACT}

Purpose: To investigate the association of clinical outcomes with platelet function-guided modification in antiplatelet therapy in patients with ischemic stroke.

Results: Among 812 patients, 223 patients had aspirin nonresponse, 204 patients was modified in antiplatelet therapy after platelet function testing. Mean followup period was $4.8 \pm 1.7$ years (ranged from 1 to 6.4 years). The incidence rates of ischemic events, death, or bleeding events were not significantly different between the patients with and without antiplatelet therapy modification. However, in patients with aspirin nonresponse, antiplatelet therapy modification was associated with decreased ischemic events (hazard ratio, 0.67 ; 95\% confidence interval [CI], $0.62-$ $0.97 ; P=0.01$ ) and ischemic stroke (hazard ratio, $0.70 ; 95 \% \mathrm{CI}, 0.63-0.98 ; P=0.03$ ) compared with no modification in antiplatelet therapy.

Conclusions: In patients with aspirin nonresponse, platelet function-guided modification in antiplatelet therapy after an ischemic stroke was associated with significantly lower rate of ischemic events. The platelet function testing may be useful to guide antiplatelet therapy modification.

Methods: This is a retrospective, multicentre study. From August 2010 to December 2014, 812 patients with ischemic stroke underwent platelet function testing using platelet aggregation. Antiplatelet therapy modification was defined as any change in antiplatelet therapy after testing, including increasing aspirin dosage, adding an additional antiplatelet agent to aspirin, or switching to a more potent antiplatelet agent. The primary outcome was ischemic events. Secondary outcomes included death and bleeding events. Clinical outcomes were compared between patients with and without antiplatelet therapy modification using univariate and propensity score-adjusted analyses.

\section{INTRODUCTION}

Stroke is a leading cause of mortality and disability [1]. In China, the age-standardized incidence rates per 100,000 person years of overall first-ever stroke were 135.0-270.0, the death rate of stroke was $11.4-15.4 \%$ during 1 year poststroke $[2,3]$. There are approximately 3 million new stroke cases every year in China, with ischemic stroke accounting for $78.9 \%$ of all stroke [2]. The risk of recurrent stroke is very high after ischemic stroke [4]. After an ischemic stroke or transient ischemic attack (TIA) of arterial origin, antiplatelet therapy, such as aspirin or clopidogrel is currently recommended to reduce the risk of recurrent ischemic events $[5,6]$. However, the response to aspirin is variable $[7,8]$. The prevalence of aspirin nonresponse ranges from 5\% to $60 \%$ $[9,10]$. Our previous studies showed that nonresponse to 
aspirin in patients with ischemic stroke is associated with an increased risk of recurrence ischemic stroke (RIS) and worse functional status $[8,11]$.

Despite aspirin nonresponse signifying a risk factor for adverse events, there are no widely accepted standardized treatment recommendations for these patients. Increasing the dose of aspirin might reduce the rate of aspirin nonresponse, and prevent occurrence of vascular events $[12,13]$, but this may increase the risk of a hemorrhagic event [14]. Adding an additional antiplatelet agent combination therapy may be useful. The Clopidogrel in High-Risk Patients with Acute Nondisabling Cerebrovascular Events (CHANCE) trial showed that the combination of clopidogrel and aspirin for the first 21 days is superior to aspirin alone for reducing the risk of stroke in the first 90 days and does not increase the risk of hemorrhage in patients with TIA or minor stroke [15]. However, the MATCH (Management of Atherothrombosis with Clopidogrel in High-risk Patients with Recent Transient Ischaemic Attack or Ischaemic Stroke) trial found that long-term combination of clopidogrel and aspirin was not more effective than clopidogrel alone in preventing recurrent ischemic events, and the risk of life-threatening or major bleeding is increased [16]. Substitution of aspirin with other antiplatelet drugs is thought to offset the effect of antiplatelet drug resistance, and may help prevent the occurrence of vascular events [12]. In a trial of patients receiving coronary stents showed no significant improvements in clinical outcomes with platelet-function monitoring and treatment adjustment for coronary stenting [17]. Improvement in clinical outcomes by intensifying antiplatelet therapy has also not been demonstrated in patients with ischemic stroke or TIA [18, 19]. A retrospective study showed that platelet functionguided modification in antiplatelet therapy after an ischemic stroke or TIA was associated with significantly increased rates of death, ischemic events, or bleeding events [14]. However, Alberts reported that modification in antiplatelet therapy according to platelet function testing was reasonable [20]. Researchers of the latter studies maintain, however, that more data are required before any firm conclusion can be drawn.

The aim of the present study was to investigate the clinical efficacy and safety of platelet function-guided modification in antiplatelet therapy in patients with acute ischemic stroke. This may be useful to guide the precise treatment of antiplatelet drugs, and develop more effective drugs to prevent recurrent ischemic events after ischemic stroke.

\section{RESULTS}

\section{Characteristics of patients}

All patients were administered $200 \mathrm{mg}$ aspirin per day for 14 days after the onset of stroke and $100 \mathrm{mg} /$ day thereafter. Among the 812 patients, 223 patients (27.5\%) had aspirin nonresponse according to platelet function testing. Table 1 compares the parameters between patients with and without aspirin nonresponse. The rate of diabetes mellitus and the level of fasting glucose were higher in patients with aspirin nonresponse than in those with AS $(P<0.001)$. There was no significant difference in other risk factors between the two groups.

\section{Antiplatelet therapy modification}

Among the 812 patients, 204 patients (25.1\%) were modified in antiplatelet therapy after platelet function testing (154 in aspirin nonresponse group, 50 in AS group). 50 patients with AS received modification in antiplatelet theraphy, because side effects of aspirin, such as allergic to aspirin $(n=1)$, asthma $(n=2)$, gastrointestinal bleeding $(n=8)$, hematuria $(n=5)$, skin or mucosal bleeding $(n=16)$, or severe nausea and vomiting $(n=18)$.

Baseline characteristics for the patients with $(n=204)$ and without $(n=608)$ ATM were shown in Table 2. Patients who underwent ATM were older, had higher platelet aggregation with AA or ADP compared with patients without ATM. Aspirin nonresponse was significantly higher in patients with ATM compared with patients without any modification.

The ATM after platelet function testing are shown in Table 3. Changing from aspirin to clopidogrel ( $n=126$, $61.8 \%$ ) was the most common modifications. Clopidogrel was added to aspirin in 37 patients (18.1\%). 23 patients $(11.3 \%)$ were changed from aspirin to cilostazol. 18 patients $(8.8 \%)$ were increased the aspirin dosage.

In aspirin nonresponders $(n=223)$, antiplatelet therapy was modified in 154 patients by changing from aspirin to clopidogrel $(n=97)$, adding clopidogrel to aspirin $(n=32)$, changing from aspirin to cilostazol $(n=15)$, increasing the aspirin dosage $(n=10) .69$ aspirin non-responders did not receive ATM, because they were not willing to receive ATM.

\section{Clinical outcomes}

Clinical follow-up was available for all patients with a mean follow-up period of $4.8 \pm 1.7$ years (ranged from 1 to 6.4 years). Ischemic events occurred in 181 (22.3\%) patients (120 had ischemic stroke, 37 had TIA and 24 had MI). Bleeding events occurred in $83(10.2 \%)$ patients. The incidence rates of is-chemic events, bleeding events, and death were not significantly different between the patients who underwent ATM compared with patients without modification (all $P>0.05$, Table 4). There were also no significant differences in incidence rates of ischemic events and death among the different ATM (Supplementary Table 1). With regard to the patients in whom clopidogrel was added, the rate of bleeding was significantly higher than the patients without modification (27.0\% [10/37] versus 9.9\% [60/608], 
Table 1: Baseline characteristics of patients with and without aspirin nonresponse

\begin{tabular}{|c|c|c|c|}
\hline Parameter & Aspirin nonresponser $n=223$ & Aspirin sensitivity $n=589$ & $P$ value* \\
\hline Age (years) & $70.7 \pm 12.8$ & $70.1 \pm 11.4$ & 0.76 \\
\hline $\operatorname{Men}(n, \%)$ & $107(48.0)$ & $317(53.8)$ & 0.24 \\
\hline Body mass index $\left(\mathrm{kg} / \mathrm{m}^{2}\right)$ & $24.6 \pm 3.4$ & $24.1 \pm 3.5$ & 0.91 \\
\hline Current smoking $(n, \%)$ & $63(28.3)$ & $172(29.2)$ & 0.98 \\
\hline Hypertension $(n, \%)$ & $166(74.5)$ & $421(71.5)$ & 0.43 \\
\hline Diabetes mellitus $(n, \%)$ & $79(35.4)$ & $93(15.8)$ & $<0.001$ \\
\hline Previous MI $(n, \%)$ & $6(2.7)$ & $10(1.7)$ & 0.42 \\
\hline NIHSS score at enrollment & $5.9 \pm 1.8$ & $5.8 \pm 1.9$ & 0.89 \\
\hline Hyperlipidemia $(n, \%)$ & $188(84.3)$ & $478(81.2)$ & 0.52 \\
\hline Fasting glucose $(\mathrm{mmol} / \mathrm{L})$ & $7.2 \pm 2.3$ & $6.5 \pm 2.5$ & $<0.001$ \\
\hline Platelet count $\left(\times 10^{9} / \mathrm{L}\right)$ & $193.2 \pm 28.8$ & $196.5 \pm 30.7$ & 0.87 \\
\hline \multicolumn{4}{|l|}{ Stroke subtype } \\
\hline Atherothrombotic $(n, \%)$ & $139(62.3)$ & $350(59.4)$ & 0.51 \\
\hline Small artery disease $(n, \%)$ & $84(37.7)$ & $239(40.6)$ & 051 \\
\hline \multicolumn{4}{|l|}{ Previous treatment $(n, \%)$} \\
\hline Antihypertensive drugs & $97(43.5)$ & $263(44.7)$ & 0.88 \\
\hline Hypoglycemic drugs & $52(23.3)$ & $92(15.6)$ & 0.014 \\
\hline Statins & $35(15.7)$ & $99(16.8)$ & 0.74 \\
\hline Aspirin & $50(22.4)$ & $141(23.9)$ & 0.73 \\
\hline
\end{tabular}

MI, myocardial infarction; NIHSS, National Institutes of Health Stroke Scale.

$P<0.001)$ or than the patients with modifications of changing from aspirin to clopidogrel or cilostazol (Supplementary Table 1). Retesting platelet function at 10 days after antiplatelet therapy modification was performed in 105 patients $(51.5 \%)$. In patients with aspirin nonresponse, $76 \%$ were responsive by adding clopidogrel, $52 \%$ were responsive by changing from aspirin to clopidogrel or cilostazol, and $41 \%$ were responsive by increasing the aspirin dosage.

In patients who were nonresponsive to aspirin $(n=223)$, ischemic events occurred in $51(22.9 \%)$ patients (35 had ischemic stroke, 9 had TIA and 7 had MI). There were no significant differences in baseline characteristics between the patients with $(n=154)$ and without $(n=69)$ ATM for the aspirin nonresponse subgroup (Table 5). However, the patients with ATM compared with the patients without modification were associated with decreased ischemic events (18.2\% versus $33.3 \%, P=0.02$, Table 5), which was primarily due to a decrease in ischemic stroke (11.7\% versus $24.6 \%, P=0.008$, Table 5). Kaplan-Meier estimates of cumulative freedom from ischemic event (logrank $P<0.001$, Figure 1A), and ischemic stroke (log-rank $P=0.006$, Figure 1B) were significantly lower in patients without ATM compared with patients who underwent modification in aspirin non-responders. However, there were no significant differences in incidence rates of bleeding events and death between the two groups (Table 5).

In patients with AS $(n=589)$, ischemic events occurred in $130(22.1 \%)$ patients ( 85 had ischemic stroke, 28 had TIA and 17 had MI). There were no significant differences in clinical outcomes between the AS patients with $(n=50)$ and without $(n=539)$ ATM (all $P>0.05$, Supplementary Table 2).

With regard to aspirin non-response patients and AS patients who did not receive ATM ( $n=69$ and $n=$ 539 , respectively), the incidence rates of ischemic events and ischemic stroke in patients with aspirin non-response were significantly higher than patients with AS $(P=0.03$ and $P=0.02$, respectively, Table 6$)$. However, there were no significant differences in incidence rates of bleeding events and death between the two groups (Table 6).

In patients with aspirin nonresponse, the unadjusted and propensity score-adjusted hazard ratios for clinical outcomes with and without modification of antiplatelet therapy are shown in Table 7. With propensity score adjustment, ATM was associated with lower rates of ischemic event (hazard ratio, 0.67 ; 95\% confidence interval (CI), $0.62-$ $0.97 ; P=0.01$ ) or ischemic stroke (hazard ratio, $0.70 ; 95 \%$ CI, 0.63-0.98; $P=0.03$ ) compared with no modification. No significant differences were seen in the propensity scoreadjusted individual rates of death, or bleeding events between the two groups. In additional analyses performed after propensity score matching of patients in the ATM $(n=72)$ and no modification $(n=72)$ groups, rates of ischemic event and ischemic stroke remained significantly lower in the ATM group (hazard ratio, 0.69; 95\% CI, 0.57-0.94; $P=0.02$, and hazard ratio, $0.67 ; 95 \% \mathrm{CI}, 0.62-0.97 ; P=0.03$, respectively). No significant difference in death, or bleeding events was seen between the 2 matched groups. 
Table 2: Baseline characteristics of patients with and without antiplatelet therapy modification

\begin{tabular}{|c|c|c|c|}
\hline & \multicolumn{2}{|c|}{ Antiplatelet Therapy Modification } & \multirow{2}{*}{$P$ value } \\
\hline & Yes $(n=204)$ & No $(n=608)$ & \\
\hline Age (years) & $71.8 \pm 11.6$ & $67.1 \pm 13.6$ & $<0.001$ \\
\hline $\operatorname{Men}(n, \%)$ & $106(51.9)$ & $318(52.3)$ & 0.99 \\
\hline Diabetes mellitus $(n, \%)$ & $50(24.5)$ & $122(20.1)$ & 0.18 \\
\hline Hypertension $(n, \%)$ & $152(74.5)$ & $435(71.5)$ & 0.42 \\
\hline Body mass index $\left(\mathrm{kg} / \mathrm{m}^{2}\right)$ & $24.5 \pm 5.2$ & $23.9 \pm 4.9$ & 0.15 \\
\hline Current smoker $(n, \%)$ & $68(33.3)$ & $167(27.5)$ & 0.12 \\
\hline Previous MI $(n, \%)$ & $6(2.9)$ & $10(1.6)$ & 0.26 \\
\hline Hyperlipidemia $(n, \%)$ & $171(83.8)$ & $495(81.4)$ & 0.43 \\
\hline Admission NIHSS & $5.93 \pm 1.8$ & $5.86 \pm 1.9$ & 0.64 \\
\hline $\begin{array}{l}\text { Stroke subtype } \\
\text { Atherothrombotic }(n, \%) \\
\text { Small artery disease }(n, \%)\end{array}$ & $\begin{array}{c}127(62.3) \\
77(37.7)\end{array}$ & $\begin{array}{l}362(59.5) \\
246(40.5)\end{array}$ & $\begin{array}{l}0.49 \\
0.49\end{array}$ \\
\hline $\begin{array}{l}\text { Previous treatment }(n, \%) \\
\text { Antihypertensive drugs } \\
\text { Hypoglycemic drugs } \\
\text { Statins } \\
\text { Aspirin }\end{array}$ & $\begin{array}{l}89(43.6) \\
39(19.1) \\
32(15.7) \\
46(22.5)\end{array}$ & $\begin{array}{l}271(44.6) \\
105(17.3) \\
102(16.8) \\
145(23.8)\end{array}$ & $\begin{array}{l}0.83 \\
0.56 \\
0.72 \\
0.71\end{array}$ \\
\hline $\begin{array}{l}\text { In-hospital treatment }(n, \%) \\
\text { Antihypertensive drugs } \\
\text { Hypoglycemic drugs } \\
\text { Statins } \\
\text { Thrombolysis }\end{array}$ & $\begin{array}{c}170(83.3) \\
65(31.9) \\
200(98.0) \\
4(2.0)\end{array}$ & $\begin{array}{c}486(79.9) \\
169(27.8) \\
598(98.4) \\
16(2.6)\end{array}$ & $\begin{array}{l}0.33 \\
0.28 \\
0.76 \\
0.61\end{array}$ \\
\hline \multicolumn{4}{|l|}{ Platelet function testing } \\
\hline Aggregation with AA, \% & $26.8 \pm 10.2$ & $20.1 \pm 8.7$ & $<0.001$ \\
\hline Aggregation with ADP, \% & $58.4 \pm 18.6$ & $47.6 \pm 16.4$ & $<0.001$ \\
\hline Aspirin nonresponse & $154(75.5)$ & $69(11.3)$ & $<0.001$ \\
\hline Aspirin sensitivity & $50(24.5)$ & $539(88.7)$ & $<0.001$ \\
\hline Follow-up period (years) & $4.7 \pm 1.6$ & $4.8 \pm 1.7$ & 0.42 \\
\hline
\end{tabular}

MI, myocardial infarction; NIHSS, national institutes of health stroke scale; AA, arachidonic acid; ADP, adenosine diphosphate.

Table 3: Modification in antiplatelet therapy after platelet function testing

\begin{tabular}{lc}
\hline Modification in Antiplatelet Therapy & $\boldsymbol{n}=\mathbf{2 0 4}$ \\
\hline Changed from aspirin to clopidogrel & $126(61.8 \%)$ \\
Changed from aspirin to cilostazol & $23(11.3 \%)$ \\
Increased aspirin & $18(8.8 \%)$ \\
Added clopidogrel to aspirin & $37(18.1 \%)$ \\
\hline
\end{tabular}

\section{DISCUSSION}

In present study, all patients underwent platelet function testing, antiplatelet therapy was modified in 204 patients after platelet function testing. The incidence rates of ischemic events, death, bleeding events were not significantly different between the patients who underwent ATM compared with no modification. However, in patients with aspirin nonresponse, antiplatelet therapy modification was associated with decreased ischemic events and ischemic stroke compared with no modification.

The prevalence of aspirin nonresponse was $27.5 \%$ in present study, and was similar to the prevalence reported in our previous studies $[8,11,21]$ and some other studies $[9,22]$. A recent systematic review and meta-analysis showed that the prevalence of high on-treatment of platelet 
Table 4: Clinical outcomes in patients with or without antiplatelet therapy modification

\begin{tabular}{|c|c|c|c|}
\hline \multirow{2}{*}{ Variable } & \multicolumn{2}{|c|}{ Antiplatelet Therapy Modification } & \multirow{2}{*}{$P$ value } \\
\hline & Yes $(n=204)$ & No $(n=608)$ & \\
\hline Ischemic events $(n, \%)$ & $43(21.1)$ & $138(22.7)$ & 0.71 \\
\hline Ischemic stroke $(n, \%)$ & $29(14.2)$ & $91(15.0)$ & 0.82 \\
\hline Transient ischemic attack $(n, \%)$ & $8(3.9)$ & $29(4.8)$ & 0.68 \\
\hline Myocardial infarction $(n, \%)$ & $6(2.9)$ & $18(3.0)$ & 0.99 \\
\hline Any bleeding event & $23(11.3)$ & $60(9.9)$ & 0.61 \\
\hline GUSTO minor $(n, \%)$ & $12(5.9)$ & $31(5.1)$ & 0.72 \\
\hline GUSTO moderate $(n, \%)$ & $8(3.9)$ & $21(3.5)$ & 0.76 \\
\hline GUSTO severe $(n, \%)$ & $3(1.5)$ & $8(1.3)$ & 0.94 \\
\hline Gastrointestinal bleeding (n, \%) & $12(5.9)$ & $41(6.7)$ & 0.72 \\
\hline Intracerebral hemorrhage (n, \%) & $2(1.0)$ & $7(1.2)$ & 0.98 \\
\hline Death $(n, \%)$ & $7(3.4)$ & $19(3.1)$ & 0.84 \\
\hline
\end{tabular}

GUSTO, Global Use of Strategies to Open Occluded Coronary Arteries.

Table 5: Baseline characteristics and clinical outcomes in aspirin non-responders

\begin{tabular}{|c|c|c|c|}
\hline \multirow{2}{*}{ Variable } & \multicolumn{2}{|c|}{ Antiplatelet Therapy Modification } & \multirow{2}{*}{$P$ value } \\
\hline & Yes $(n=154)$ & No $(n=69)$ & \\
\hline Age (years) & $70.9 \pm 11.9$ & $70.4 \pm 10.8$ & 0.76 \\
\hline $\operatorname{Men}(n, \%)$ & $70(45.5)$ & $37(53.6)$ & 0.27 \\
\hline Diabetes mellitus $(n, \%)$ & $54(35.4)$ & $25(36.2)$ & 0.91 \\
\hline Hypertension $(n, \%)$ & $116(75.3)$ & $50(72.5)$ & 0.67 \\
\hline Current smoking $(n, \%)$ & $46(29.9)$ & $17(24.6)$ & 0.43 \\
\hline Previous MI (n, \%) & $4(2.6)$ & $2(2.9)$ & 0.99 \\
\hline NIHSS score at enrollment & $5.8 \pm 2.3$ & $5.9 \pm 2.1$ & 0.65 \\
\hline Hyperlipidemia $(n, \%)$ & $127(82.5)$ & $61(88.4)$ & 0.26 \\
\hline Fasting glucose (mmol/L) & $7.1 \pm 2.2$ & $7.3 \pm 2.4$ & 0.56 \\
\hline Platelet count $\left(\times 10^{9} / \mathrm{L}\right)$ & $189.2 \pm 25.8$ & $195.2 \pm 29.2$ & 0.14 \\
\hline \multicolumn{4}{|l|}{ Stroke subtype } \\
\hline Atherothrombotic $(n, \%)$ & $95(61.7)$ & $44(63.8)$ & 0.78 \\
\hline Small artery disease $(n, \%)$ & $59(38.3)$ & $25(36.2)$ & 0.78 \\
\hline Ischemic events $(n, \%)$ & $28(18.2)$ & $23(33.3)$ & 0.02 \\
\hline Ischemic stroke $(n, \%)$ & $18(11.7)$ & $17(24.6)$ & 0.008 \\
\hline Transient ischemic attack $(n, \%)$ & $6(3.9)$ & $3(4.3)$ & 0.92 \\
\hline $\operatorname{MI}(n, \%)$ & $4(2.6)$ & $3(4.3)$ & 0.48 \\
\hline Any bleeding event & $15(9.7)$ & $6(8.7)$ & 0.81 \\
\hline GUSTO minor $(n, \%)$ & $9(5.8)$ & $4(5.8)$ & 0.99 \\
\hline GUSTO moderate $(n, \%)$ & $4(2.6)$ & $1(1.4)$ & 0.64 \\
\hline GUSTO severe $(n, \%)$ & $2(1.3)$ & $1(1.4)$ & 0.99 \\
\hline Gastrointestinal bleeding $(n, \%)$ & $9(5.8)$ & $3(4.3)$ & 0.99 \\
\hline Intracerebral hemorrhage $(n, \%)$ & $1(0.6)$ & $1(1.4)$ & 0.52 \\
\hline Death $(n, \%)$ & $4(2.6)$ & $3(4.3)$ & 0.67 \\
\hline
\end{tabular}

MI, myocardial infarction; NIHSS, National Institutes of Health Stroke Scale; GUSTO, Global Use of Strategies to Open Occluded Coronary Arteries. 
reactivity (HTPR) on aspirin was 23\% (95\% CI: 20-28\%), and the patients with HTPR had a significantly higher risk for ischemic stroke recurrence (relative risk $=1.81,95 \%$ CI: $1.30-2.52 ; P<0.001$ ) [23]. The finding is consistent with our present study. The mechanisms associated with aspirin nonresponse are complex and mutilfactorial, such as noncompliance, diabetes mellitus, reduced absorption, the biosynthesis of thromboxane A2 from pathways not inhibited by aspirin as well as alternative pathways involved in platelet activation not blocked by aspirin
[9, 11, 21, 22]. In present study, our results also showed that the diabetes mellitus was associated with aspirin nonresponse. Patients with diabetes mellitus were usually associated with hyperlipidemia, chronic inflammation, platelet dysfunction and endothelial dysfunction, and these result in poor responsiveness to aspirin [24]. Other potential mechanisms for aspirin nonresponse in patients with diabetes mellitus may include increased circulating ADP, calcium, or esterase levels, as well as platelet turnover, the expression of $\mathrm{P} 2 \mathrm{Y} 12$ receptors, or the
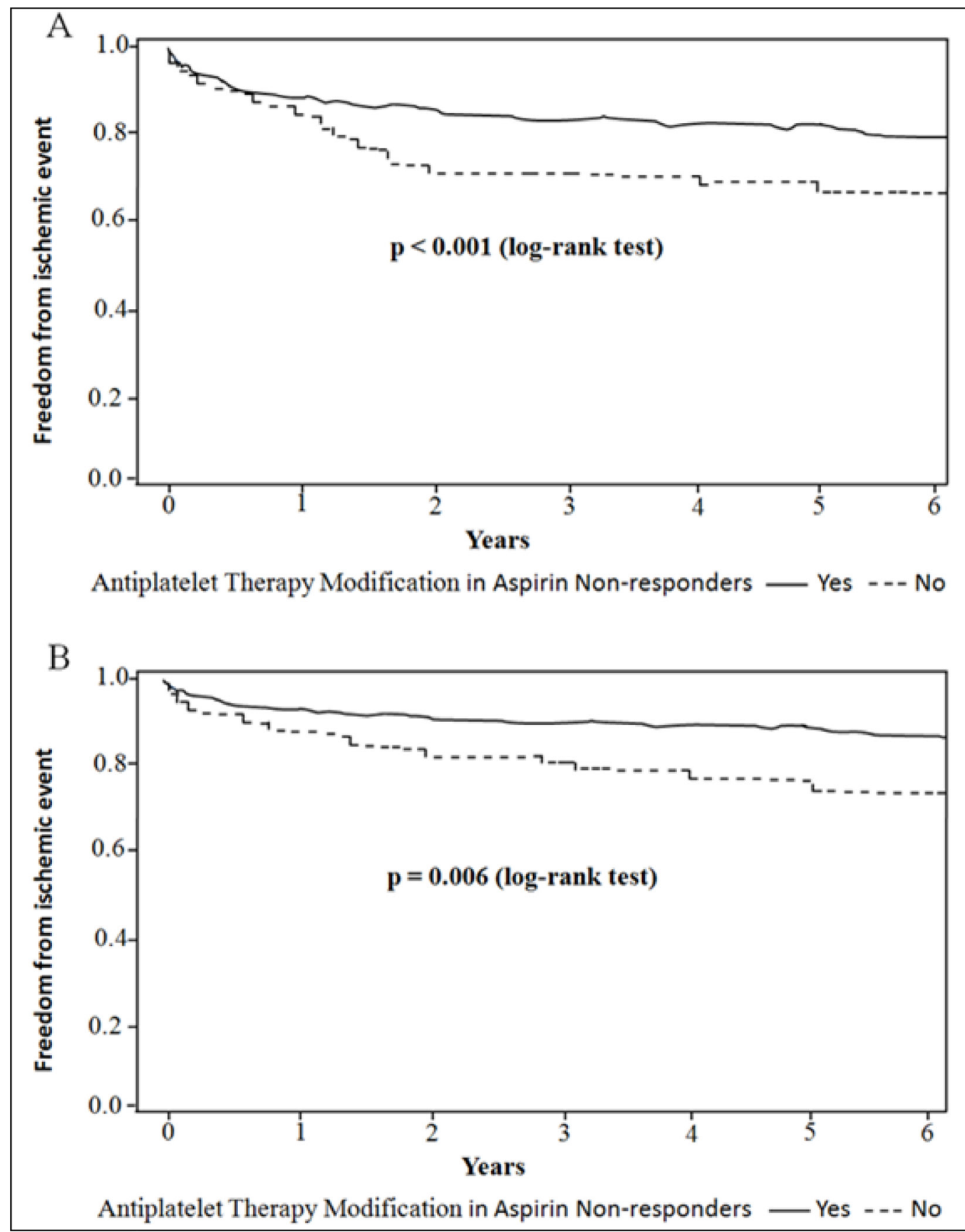

Figure 1: Kaplan-Maier analysis of clinical outcomes associated with and without modifying antiplatelet therapy after platelet function testing in aspirin non-responders. Freedom from $(\mathbf{A})$ ischemic event; $(\mathbf{B})$ ischemic stroke are compared between patients with and without antiplatelet therapy modification with a log-rank test and its associated $P$ value. 
Table 6: Clinical Outcomes between aspirin non-response patients and aspirin sensitive patients who did not receive the modified antiplatelet therapy

\begin{tabular}{lccc}
\hline Variable & aspirin sensitive patients $(\boldsymbol{n}=\mathbf{5 3 9})$ & aspirin non-responders $(\boldsymbol{n}=\mathbf{6 9})$ & $\boldsymbol{P}$ value \\
\hline Ischemic events $(n, \%)$ & $115(21.3)$ & $23(33.3)$ & 0.03 \\
Ischemic stroke $(n, \%)$ & $74(13.7)$ & $17(24.6)$ & 0.02 \\
Transient ischemic attack $(n, \%)$ & $26(4.8)$ & $3(4.3)$ & 0.88 \\
Myocardial infarction $(n, \%)$ & $15(3.3)$ & $3(4.3)$ & 0.43 \\
Any bleeding event & $54(10.0)$ & $6(8.7)$ & 0.74 \\
GUSTO minor $(n, \%)$ & $27(5.0)$ & $4(5.8)$ & 0.81 \\
GUSTO moderate $(n, \%)$ & $20(3.7)$ & $1(1.4)$ & 0.36 \\
GUSTO severe $(n, \%)$ & $7(1.3)$ & $1(1.4)$ & 0.98 \\
Gastrointestinal bleeding $(n, \%)$ & $38(7.1)$ & $3(4.3)$ & 0.45 \\
Intracerebral hemorrhage $(n, \%)$ & $6(1.1)$ & $1(1.4)$ & 0.86 \\
Death $(n, \%)$ & $16(3.0)$ & $3(4.3)$ & 0.56 \\
\hline
\end{tabular}

GUSTO, Global Use of Strategies to Open Occluded Coronary Arteries.

Table 7: Unadjusted and adjusted hazards ratios (HRs) for clinical outcomes with and without antiplatelet therapy modification in aspirin non-responders

\begin{tabular}{lcccccccc}
\hline \multirow{2}{*}{ Clinical Outcome e } & \multicolumn{2}{c}{ Unadjusted } & & \multicolumn{2}{c}{ Propensity Score Adjusted } & & \multicolumn{2}{c}{ Propensity Score Matched } \\
\cline { 2 - 3 } & HR (95\% CI) & $\boldsymbol{P}$ value & & HR (95\% CI) & $\boldsymbol{P}$ value & & HR (95\% CI) & $\boldsymbol{P}$ value \\
\hline Ichemic events & $0.63(0.51-0.88)$ & $<0.001$ & & $0.67(0.62-0.97)$ & 0.01 & & $0.69(0.57-0.94)$ & 0.02 \\
Ischemic stroke & $0.66(0.56-0.98)$ & 0.004 & & $0.70(0.63-0.98)$ & 0.03 & & $0.67(0.62-0.97)$ & 0.03 \\
Bleeding event & $1.33(0.78-4.38)$ & 0.61 & & $1.39(0.89-4.45)$ & 0.69 & & $1.25(0.87-4.85)$ & 0.17 \\
Death & $1.35(0.64-3.85)$ & 0.65 & & $1.41(0.91-4.07)$ & 0.64 & & $1.16(0.88-4.29)$ & 0.41 \\
\hline
\end{tabular}

upregulation of other platelet activation pathways [25]. Thus, intensive antiplatelet therapy may be important in diabetic patients sustaining an ischemic stroke.

Several studies have shown that nonresponse to aspirin is associated with more frequent neurologic deterioration, less frequent clinical improvement, and greater risk of recurrent ischemic events in patients with acute ischemic stroke [8, 11, 26, 27]. Alberts et al suggested that modification in antiplatelet therapy according to platelet function testing was reasonable [20]. However, there are some debates about the clinical efficacy and safety of platelet function-guided modification in antiplatelet therapy in patients with acute ischemic stroke. Our current results showed that the incidence rates of ischemic events, death, bleeding events were not significantly different between the patients with and without ATM after ischemic stroke, and were consistent with previous studies $[14,17]$. The majority of aspirin nonresponse reported in the literature may be the result of poor adherence and clinical factors that predict aspirin nonresponse are not consistent between different platelet function tests [28]. Thus, platelet function testing is not recommended in the current guidelines for management of ischemic stroke [5].

Nevertheless, key observation were identified in the present study via stratified analyses, stratified analyses revealed that the incidence rates of ischemic events and ischemic stroke were significantly higher in patients with aspirin non-response than patients with AS who did not receive ATM, and ATM was associated with decreasing ischemic events and ischemic stroke compared with no modification in patients with aspirin nonresponse. However, our findings were inconsistent with other results $[14,17]$. Collet et al [17] reported that there were no significant improvements in clinical outcomes with platelet-function monitoring and treatment adjustment for coronary stenting. Depta et al [14] showed that modification in antiplatelet therapy after an ischemic stroke or TIA was associated with significantly increased rates of death, ischemic events, or bleeding compared with no modification. However, only 324 patients with ischemic stroke or TIA were enrolled in the retrospective study, the small samples is may be a important cause for the conflicting results.

In patients with aspirin nonresponse, preventing recurrent ischemic stroke after ischemic stroke with aspirin therapy remains a challenge. There are no standardized treatment recommendations for these patients with aspirin nonresponse. In present study, ATM included changing from aspirin to clopidogrel or cilostazol $(n=149)$, adding clopidogrel to aspirin $(n=37)$, and increasing the aspirin dosage $(n=18)$. There were no significant differences in incidence rates of ischemic events and death among 
the different ATM. However, the rate of bleeding was significantly higher in patients with modification of adding clopidogrel to aspirin or increasing the aspirin dosage than patients with modifications of changing from aspirin to clopidogrel or cilostazol, or without modification. Increasing the dose of aspirin might reduce the incidence of aspirin nonresponse, and prevent occurrence of vascular events $[12,13]$, but higher doses of aspirin may increase the risk of a hemorrhagic event [14]. Dual antiplatelet therapy with aspirin and clopidogrel for the first 21 days or 30 days in patients with acute ischemic stroke can reduce the risk of stroke, and improve 6-month outcome [15, 29, 30]. However, long-term combination of clopidogrel and aspirin was not more effective than clopidogrel alone in preventing recurrent ischemic events, and the risk of life-threatening or major bleeding is increased [16]. Our results also showed the rate of bleeding was higher in patients in whom clopidogrel was added or dose of aspirin was increased than patients without modification. Thus, increasing the dose of aspirin or longterm dual antiplatelet therapy with aspirin and clopidogrel for the secondary prevention of ischemic stroke were inadequate for these patients. Substitution of aspirin with another antiplatelet drug (like clopidogrel or cilostazol) is thought to optimize regime, and may help prevent the occurrence of vascular events [31, 32]. The Clopidogrel versus Aspirin in Patients at Risk of Ischemic Events (CAPRIE) trial demonstrated that clopidogrel is more effective than aspirin in reducing the combined risk of ischemic stroke, MI, or vascular death in patients with atherosclerotic vascular disease [33]. A meta-analysis to estimate the efficacy of antiplatelet agents for secondary prevention of recurrent stroke demonstrated that cilostazol was significantly more efficient than other antiplatelet agents in Asian patients [32]. These were consistent with our current findings. However, further randomizedcontrolled trials are needed to validate our findings.

The risk of recurrent stroke is very high after ischemic stroke, and aspirin is recommended to reduce the risk of recurrent ischemic events in patients with ischemic stroke. However, the response to aspirin is variable. Our previous studies showed that nonresponse to aspirin in patients with ischemic stroke is associated with an increased risk of recurrence ischemic stroke and worse functional status, and platelet function testing may be useful as a marker of increased risk for recurrent events after ischemic stroke $[8,11]$. In presents study, our results revealed that ATM after platelet function testing was associated with decreasing ischemic events and ischemic stroke in patients with aspirin nonresponse. Up to date, few studies assessed the efficacy and safety of modifications in antiplatelet therapy according to platelet function testing in patients with acute ischemic stroke. The results of our study indicate that platelet function testing may be useful to guide ATM and optimize clinical outcomes in patients with aspirin nonresponse. Our these findings could be useful to guide the precise treatment of antiplatelet drugs, decrease the risk of recurrent ischemic events, improve functional status, and develop more effective drugs to prevent recurrent ischemic events after ischemic stroke.

Several important limitations of our study should be considered. First, our study is retrospective and observational, and this may limit the generalizability of the results. Additionally, the diverse modifications in antiplatelet regimens used after platelet function testing were at the physician's discretion. It is unknown what clinical factors led each physician to decide which therapeutic regimen to use after platelet function testing, thus making it very difficult to control for selection bias. Second, several laboratory tests are used to assess the response to aspirin, including LTA, bleeding time, platelet function analyzer-100, the VerifyNow Aspirin system. Each method has its own advantages and disadvantages [33]. However, platelet aggregation was only measured using the LTA in this study. Third, retesting platelet function after antiplatelet therapy modification was only performed in 105 patients, the infrequency of retesting limited our ability to determine if responsiveness after antiplatelet therapy modification resulted in any clinical benefit. Fourth, although careful analysis was performed to account for any differences between patients with and without antiplatelet therapy modification, unknown confounders may have contributed to the differences in clinical outcomes between both groups. Furthermore, the current study may also have possible bias due to the threecenter, relative small sample size. Therefore, our findings must be validated in multi-center, larger sample size, and randomized-controlled trials.

\section{CONCLUSIONS}

In patients with aspirin nonresponse, antiplatelet therapy modification was associated with decreased ischemic events and ischemic stroke compared with no modification. The results indicate that platelet function testing is may be useful to guide antiplatelet therapy modification, and optimize clinical outcomes, although our results should be interpreted with caution given the possible confounding role of selection bias. Randomizedcontrolled trials are needed to determine if a platelet function-guided approach is beneficial and safe to prevent recurrent events after ischemic stroke in future.

\section{MATERIALS AND METHODS}

\section{Study population}

This retrospective, multi-centre study was jointly conducted by the People's Hospital of Deyang City, the second, and third Affiliated Hospital of Wenzhou Medical University. The study protocol was approved by the Ethics Committee at the participating hospitals. Written informed 
consent was obtained from each patient. Institutional Review Board approval was obtained on January 31, 2016.

We consecutively enrolled patients who underwent a first-ever ischemic stroke and were admitted to the participating hospitals within $72 \mathrm{~h}$ of the onset of stroke between August 2010 and December 2014. The inclusion criteria were: (1) age $\geq 40$ years old; (2) all patients underwent platelet function testing; (3) all patients were receiving aspirin monotherapy before the platelet function testing; (4) absence of endovascular or surgical treatment for stroke. Exclusion criteria were: (1) cerebral embolism or undetermined etiologies of ischemic stroke; (2) patients whose antiplatelet therapy was decreased or who had warfarin added during observational phase; (3) loss to follow-up. A total of 883 patients met above inclusion criteria and exclusion criteria. However, 71 patients declined to participate this study. Thus, 812 patients were enrolled. The overall response rate was approximately 92\% (812/883) [92.6\% (287/310) in the People's Hospital of Deyang City, 91.2\% (207/227) and 91.9\% (318/346) in the second and third Affiliated Hospital of Wenzhou Medical University, respectively].

All enrolled patients received standard therapies based on the guidelines for the prevention of stroke in patients with stroke and TIA [5]. All patient's data were obtained through the electronic medical record system and/or paper charts and were independently verified by the authors. Hypertension was defined as the mean of three independent measures of BP $\geq 140 / 90 \mathrm{mmHg}$ or the use of antihypertensive drugs. Diabetes mellitus was diagnosed by any one or a combination of fasting glucose level $>7.8$ $\mathrm{mmol} / \mathrm{L},>11.1 \mathrm{mmol} / \mathrm{L} 2 \mathrm{~h}$ after oral glucose challenge, and use of hypoglycemic drugs. Dyslipidemia was defined as $\mathrm{TC}>200 \mathrm{mg} / \mathrm{dL}, \mathrm{TG}>180 \mathrm{mg} / \mathrm{dL}$ or use of lipidlowering medication. Cigarette smoking was defined as smoking of at least one cigarette per day for more than 1 year [34].

\section{Platelet function testing and definition of antiplatelet resistance}

Blood samples were collected at 7-10 days after aspirin therapy. Platelet function was measured by light transmittance aggregometry (LTA). The procedures and consistency tests were performed as described in our previous studies $[8,10,11]$. In the present study, aspirin resistance (AR) was defined as a mean platelet aggregation $\geq 20 \%$ with $0.5 \mathrm{mM}$ arachidonic acid (AA) and $\geq 70 \%$ with $10 \mu \mathrm{M}$ adenosine diphosphate (ADP) at 7-10 days after aspirin therapy. Patients who meet only 1 of the above 2 criteria are defined as aspirin semi-resistance. For the purposes of our study, aspirin non-response was defined as any patient meeting either criteria and currently on aspirin [11]. Otherwise, patients were considered aspirin sensitive (AS).

\section{Definition of antiplatelet therapy modification}

The definition used for antiplatelet therapy modification (ATM) was any change in the patient's antiplatelet regimen within 24 hours after the platelet function testing result was made available. Change in antiplatelet therapy was defined as any increasing the dosage of aspirin (200 mg/d increase to $300 \mathrm{mg} / \mathrm{d})$, adding an additional antiplatelet agent to aspirin (add clopidogrel to aspirin), or switching to a more potent antiplatelet agent (eg, change from aspirin to clopidogrel or cilostazol). The following patients needed to receive ATM: (1) aspirin non-responders, and these non-responders were willing to receive ATM; (2) side effects of aspirin, such as allergic to aspirin, gastrointestinal bleeding, skin or mucosal bleeding, and severe nausea and vomiting. For the patients with side effects of aspirin, aspirin was switched to clopidogrel or cilostazol. For aspirin non-responders, one following ATM was selected: (1) increasing the dosage of aspirin; (2) adding an additional antiplatelet agent to aspirin; (3) switching to a more potent antiplatelet agent. This was a multi-center, retrospective study, not a randomized-controlled trial. In addition, up to date, there are no standardized treatment recommendations for the aspirin non-responders. Thus, the diverse modifications in antiplatelet regimens used after platelet function testing were at the discretion of the treating physician in this study.

\section{Assessment of clinical outcomes}

The primary outcome of the study was ischemic events. Ischemic events were defined as an ischemic stroke, TIA, myocardial infarction (MI). Ischemic stroke was defined as any non-hemorrhagic or embolic stroke with loss of neurological function caused by an ischemic event with residual symptoms at least 24 hours after onset, where as TIA was defined as loss of neurological function without residual deficit at 24 hours. MI was defined as the presence of at least two of these criteria: prolonged angina $>30 \mathrm{~min}$; total creatinine kinase isoenzyme elevation more than twice the upper limit of normal; electrocardiographic evidence of infarction.

Secondary outcomes included death and bleeding events. Death was defined as all-cause mortality. Bleeding events were defined according to the Global Use of Strategies to Open Occluded Coronary Arteries (GUSTO) bleeding classification [35]. GUSTO Severe or life-threatening bleeding was defined as any intracranial hemorrhage or bleeding that causes hemodynamic compromise requiring intervention. Any bleeding that required blood transfusion in the absence of hemodynamic compromise was considered GUSTO moderate bleeding. GUSTO minor bleeding was defined as any bleeding that did not meet criteria for severe or moderate bleeding. 
Follow-up was performed by telephone interview and by reviewing the medical charts of each participant regardless of aspirin resistance status. The researchers who performed follow-up interviews were blinded to aspirin sensitivity status. Scheduled follow-up telephone calls were made after discharge to support proper compliance, answer any queries, and record complaints of any side effects. For those patients who reached at least one of the primary end points, a medical chart review was initiated to determine whether the event met the definitions described earlier. The terminal time of follow-up was December 31, 2016.

\section{Statistical analysis}

All statistical analyses were performed using SPSS 16.0 (SPSS Inc., Chicago, IL, USA). Differences between the antiplatelet therapy modification and no modification groups were analyzed by univariate methods. Categorical variables are presented as frequencies and percentages and compared using the Chi-square or Fisher's exact tests. Continuous variables are expressed as mean \pm Standard Deviation (SD) and compared using the Student's $t$-test. Survival function estimates for clinical outcomes were evaluated through Kaplan-Meier analyses. Survival curves were truncated at year 5. The log-rank test was used to identify differences between antiplatelet therapy modification and no modification groups.

Propensity scores were created for antiplatelet therapy modification and no modification groups based on patient characteristics. The following variables were used to calculate the propensity score: age, male, inpatient, smoking status, diabetes mellitus, hypertension, hyperlipidemia, prior MI, prior percutaneous coronary intervention, prior coronary artery bypass $\operatorname{graft}(\mathrm{s})$, history of aspirin, antihypertensive drugs, hypoglycemic drugs, and statins. Covariate balance between groups was evaluated by examining the Wald chi-square statistic before and after propensity score adjustment. After adjusting for propensity score, none of the variables used to create propensity score were found to be significantly different between groups. An additional analysis on matched propensity scores was conducted and standardized differences were calculated to determine covariate balance before and after matching. A Cox proportional hazards model for each outcome was created with and without propensity score adjustment. All tests were two-sided, and $P$ values of 0.05 were considered to represent statistical significance.

\section{Abbreviations}

CI, confidence interval; TIA, transient ischemic attack; RIS, recurrence ischemic stroke; TC, total cholesterol; TG, triglycerides; LTA, light transmittance aggregometry; AR, aspirin resistance; AS, aspirin sensitive; AA, arachidonic acid; ADP, adenosine diphosphate; GUSTO, Global Use of Strategies to Open Occluded Coronary Arteries; MI, myocardial infarction; HTPR, high on-treatment of platelet reactivity; NIHSS, National Institutes of Health Stroke Scale.

\section{Author contributions}

Xingyang Yi participated in the design of the study, statistical analysis, and drafted the manuscript. Jing Lin participated in statistical analysis and discussion. Chun Wang helped to draft the manuscript. Ruyue Huang carried out the molecular genetic studies, participated in the sequence alignment. Zhao Han participated in the sequence alignment. Jie Li participated in statistical analysis, and drafted the manuscript. All authors read and approved the final manuscript.

\section{ACKNOWLEDGMENTS}

None.

\section{CONFLICTS OF INTEREST}

The authors declare no conflicts of interest.

\section{FUNDING}

This study was supported in part by grants from the Deyang City Science and Technology Research Foundation (Grant No. 2014SZ035) and Scientific Research Foundation of Chengdu University of Traditional Chinese Medicine (Grant No.YYZX1510).

\section{Declarations}

\section{Ethics approval and consent to participate}

The study protocol was approved by the Ethics Committee of the People's Hospital of Deyang City and the Third Affiliated Hospital of Wenzhou Medical University. Written informed consent was obtained from each patient prior to study enrollment.

\section{Consent for publication}

Consent for publication is obtained from all participants.

\section{REFERENCES}

1. Wang YL, Wu D, Liao X, Zhang W, Zhao X, Wang YJ. Burden of stroke in china. Int J Stroke. 2007; 2: 211-213.

2. Jiang B, Wang WZ, Chen H, Hong Z, Yang QD, Wu SP, $\mathrm{Du} \mathrm{XL}$, Bao QJ. Incidence and trends of stroke and its 
subtypes in china: Results from three large cities. Stroke. 2006; 37:63-68.

3. Wei JW, Heeley EL, Wang JG, Huang Y, Wong LK, Li Z, Heritier S, Arima H, Anderson CS; ChinaQUEST Investigators. Comparison of recovery patterns and prognostic indicators for ischemic and hemorrhagic stroke in China: the ChinaQUEST (QUality Evaluation of Stroke Care and Treatment) Registry study. Stroke. 2010; 41:1877-1883.

4. Zhao D, Liu J, Wang W, Zeng Z, Cheng J, Liu J, Sun J, $\mathrm{Wu} \mathrm{Z}$. Epidemiological transition of stroke in China: twenty-one-year observational study from the SinoMONICA-Beijing Project. Stroke. 2008; 39:1668-1674.

5. Kernan WN, Ovbiagele B, Black HR, Bravata DM, Chimowitz MI, Ezekowitz MD, Fang MC, Fisher M, Furie KL, Heck DV, Johnston SC, Kasner SE, Kittner SJ, et al. Guidelines for the prevention of stroke in patients with stroke and transient ischemic attack: a guideline for healthcare professionals from the American Heart Association/American Stroke Association. Stroke. 2014; 45:2160-2236.

6. Antithrombotic Trialists' Collaboration. Collaborative meta-analysis of randomised trials of antiplatelet therapy for prevention of death, myocardial infarction, and stroke in high risk patients. BMJ. 2002; 324:71-86.

7. Greer DM. Aspirin and antiplatelet agent resistance: implications for prevention of secondary stroke. CNS Drugs. 2010; 24:1027-1040.

8. Yi X, Wang C, Liu P, Fu C, Lin J, Chen Y. Antiplatelet drug resistance is associated with early neurological deterioration in acute minor ischemic stroke in the Chinese population. J Neurol. 2016; 263:1612-1629.

9. Fong J, Cheng-Ching E, Hussain MS, Katzan I, Gupta R. Predictors of biochemical aspirin and clopidogrel resistance in patients with ischemic stroke. J Stroke Cerebrovasc Dis. 2011; 20:227-230.

10. Yi X, Zhou Q, Lin J, Chi L, Han Z. Platelet Response to Aspirin in Chinese Stroke Patients is Independent of Genetic Polymorphisms of COX-1 C50T and COX-2 G765C. J Atheroscler Thromb. 2013; 20:65-72.

11. Yi X, Zhou Q, Lin J, Chi L. Aspirin resistance in Chinese stroke patients increased the rate of recurrent stroke and other vascular events. Int J Stroke. 2013; 8:535-539.

12. Mehta SR, Tanguay JF, Eikelboom JW, Jolly SS, Joyner CD, Granger CB, Faxon DP, Rupprecht HJ, Budaj A, Avezum A, Widimsky P, Steg PG, Bassand JP, et al. Double-dose versus standard-dose clopidogrel and high-dose versus low-dose aspirin in individuals undergoing percutaneous coronary intervention for acute coronary syndromes (CURRENTOASIS 7): a randomised factorial trial. Lancet. 2010; 376:1233-1243.

13. Helgason CM, Tortorice KL, Winkler SR, Penney DW, Schuler JJ, McClelland TJ, Brace LD. Aspirin response and failure in cerebral infarction. Stroke. 1993; 24:345-350.
14. Depta JP, Fowler J, Novak E, Katzan I, Bakdash S, KottkeMarchant K, Bhatt DL. Clinical outcomes using a platelet function-guided approach for secondary prevention in patients with ischemic stroke or transient ischemic attack. Stroke. 2012; 43:2376-2381.

15. Wang Y, Wang Y, Zhao X, Liu L, Wang D, Wang C, Wang C, Li H, Meng X, Cui L, Jia J, Dong Q, Xu A, et al. Clopidogrel with aspirin in acute minor stroke or transient ischemic attack. N Engl J Med. 2013; 369:11-19.

16. Diener HC, Bogousslavsky J, Brass LM, Cimminiello C, Csiba L, Kaste M, Leys D, Matias-Guiu J, Rupprecht HJ. Aspirin and clopidogrel compared with clopidogrel alone after recent ischaemic stroke or transient ischaemic attack in high-risk patients (MATCH): randomised, double-blind, placebo-controlled trial. Lancet. 2004; 364:331-337.

17. Collet JP, Cuisset T, Rangé G, Cayla G, Elhadad S, Pouillot C, Henry P, Motreff P, Carrié D, Boueri Z, Belle L, Van Belle E, Rousseau H, et al. Bedside monitoring to adjust antiplatelet therapy for coronary stenting. N Engl J Med. 2012; 367:2100-2109.

18. Selim $\mathrm{MH}$, Molina CA. Platelet function assays in stroke management: more study is needed. Stroke. 2010; 41:2396-2397.

19. Eikelboom JW, Emery J, Hankey GJ. The use of platelet function assays may help to determine appropriate antiplatelet treatment options in a patient with recurrent stroke on baby aspirin: against. Stroke. 2010; 41:2398-2399.

20. Alberts MJ. Platelet function testing for aspirin resistance is reasonable to do: yes! Stroke. 2010; 41:2400-2401.

21. Yi X, Cheng W, Lin J, Zhou Q, Wang C. Interaction between COX-1 and COX-2 Variants Associated with Aspirin Resistance in Chinese Stroke Patients. J Stroke Cerebrovasc Dis. 2016; 25:2136-2144.

22. Hankey GJ, Eikelboom JW. Aspirin resistance. Biomed Res Int. 2016; 2016:9821767.

23. Fiolaki A, Katsanos AH, Kyritsis AP, Papadaki S, Kosmidou M, Moschonas IC, Tselepis AD, Giannopoulos S. High on treatment platelet reactivity to aspirin and clopidogrel in ischemic stroke: A systematic review and meta-analysis. J Neurol Sci. 2017; 376:112-116.

24. Habizal NH, Abdul Halim S, Bhaskar S, Wan Bebakar WM, Abdullah JM. Prevalence of aspirin resistance in diabetic patients and its associated factors. Malays J Med Sci. 2015; 22:50-57.

25. Ferreiro JL, Gomez-Hospital JA, Angiolillo DJ. Platelet abnormalities in diabetes mellitus. Diab Vasc Dis Res. 2010; 7: 251-259.

26. Jeon SB, Song HS, Kim BJ, Kim HJ, Kang DW, Kim JS, Kwon SU. Biochemical aspirin resistance and recurrent lesions in patients with acute ischemic stroke. European neurology. 2010; 64:51-57. 
27. Bugnicourt JM, Roussel B, Garcia PY, Canaple S, Lamy C, Godefroy O. Aspirin non-responder status and early neurological deterioration: A prospective study. Clin Neurol Neurosurg. 2011; 113:196-201.

28. Seok JI, Joo IS, Yoon JH, Choi YJ, Lee PH, Huh K, Bang OY. Can aspirin resistance be clinically predicted in stroke patients? Clin Neurol Neurosurg. 2008; 110:110-116.

29. Wang C, Yi X, Zhang B, Liao D, Lin J, Chi L. Clopidogrel Plus Aspirin Prevents Early Neurologic Deterioration and Improves 6-Month Outcome in Patients With Acute Large Artery Atherosclerosis Stroke. Clin Appl Thromb Hemost. 2015; 21:453-61

30. Yi X, Lin J, Wang C, Zhang B, Chi W. A comparative study of dual versus monoantiplatelet therapy in patients with acute large-artery atherosclerosis stroke. J Stroke Cerebrovasc Dis. 2014 ; 23:1975-1981.
31. CAPRIE Steering Committee. A randomised, blinded, trial of clopidogrel versus aspirin in patients at risk of ischaemic events (CAPRIE). Lancet. 1996; 348:1329-1339.

32. Wang W, Zhang L, Liu W, Zhu Q, Lan Q, Zhao J. Antiplatelet Agents for the Secondary Prevention of Ischemic Stroke or Transient Ischemic Attack: A Network Meta-Analysis. J Stroke Cerebrovasc Dis. 2016; 25:1081-1089.

33. Michelson AD, Frelinger AL 3rd, Furman MI. Current options in platelet function testing. Am J Cardiol. 2006; 98:4N-10N.

34. Yi X, Han Z, Zhou Q, Lin J, Liu P. 20-Hydroxyeicosatetraenoic Acid as a Predictor of Neurological Deterioration in Acute Minor Ischemic Stroke. Stroke. 2016; 47:3045-3047.

35. GUSTO investigators. An international randomized trial comparing four thrombolytic strategies for acute myocardial infarction. N Engl J Med. 1993; 329:673-682. 